\title{
Coulomb Effects on Electron Transport and Shot Noise in Hybrid Normal-Superconducting Metallic Structures
}

\author{
Artem V. Galaktionov and Andrei D. Zaikin \\ Forschungszentrum Karlsruhe, Institut für Nanotechnologie, 76021, Karlsruhe, Germany \\ I.E. Tamm Department of Theoretical Physics, P.N. Lebedev Physics Institute, 119991 Moscow, Russia
}

\begin{abstract}
We analyze the effect of electron-electron interactions on Andreev current and shot noise in diffusive hybrid structures composed of a normal metal attached to a superconductor via a weakly transmitting interface. We demonstrate that at voltages/temperatures below the Thouless energy of a normal metal Coulomb interaction yields a reduction of both Andreev current and its noise by a constant factor which essentially depends on the system dimensionality. For quasi-1d structures this factor is $\sim N_{\mathrm{Ch}}^{8 / g}$, where $N_{\mathrm{Ch}}$ and $g$ are respectively the number of conducting channels and dimensionless conductance of a normal metal. At voltages above the Thouless energy the interaction correction to Andreev current and shot noise acquires an additional voltage dependence which turns out to be a power-law in the quasi-1d limit.
\end{abstract}

\section{INTRODUCTION}

It is well established that low temperature charge transport through an interface between a normal metal and a superconductor (NS) is dominated by Andreev reflection ${ }^{1}$ : An electron with energy below the superconducting gap $\Delta$ enters the superconductor from the normal metal, forms a Cooper pair together with another electron, while a hole goes back into the normal metal. In addition to Andreev reflection electrons can be scattered at the interface potentials and/or impurities. The combination of "normal" and Andreev reflection mechanisms is essential for proper understanding of transport phenomena in proximity structures composed of superconducting and normal metals. This applies both to equilibrium phenomena, such as dc Josephson effect in diffusive SNS hybrids 2.3.4.5.6 and Meissner effect in NS systems 7.8 , and to non-equilibrium effects, such as dissipative transport of subgap electrons across NS interfaces $\stackrel{9}{\text {. }}$.

For weakly transmitting NS interfaces the corresponding Andreev conductance $G_{A}$ turns out to be rather small as a second order effect in the interface transmission. At the same time interplay between disorder and interference effects in the normal metal may strongly enhance Andreev conductance at sufficiently low energies $10,11.12 .13$ leading to the so-called zero-bias anomaly (ZBA) on the current-voltage characteristics. In the case of diffusive metals Andreev conductance grows as

$$
G_{A} \propto 1 / \sqrt{T}, \quad G_{A} \propto 1 / \sqrt{V}
$$

with decreasing temperature and applied voltage $V$.

What is the effect of Coulomb interaction on the Andreev conductance of NS structures? In the limit of weakly transmitting NS interfaces this question was addressed in Ref. 13 within a simple capacitive model and the Coulomb blockade of Andreev reflection was predicted at sufficiently low energies. Huck et al $\stackrel{14}{\underline{14}}$ studied the problem by modeling the effect of interactions by means of an effective electromagnetic environment with an impedance $Z(\omega)$. Similarly to the the case of normal tunnel junctions ${ }^{15.16}$, for the Ohmic environment
$Z(\omega)=R$ Coulomb interaction yields a power-law ZBA which, being combined with Eq. (1), yields 14

$$
G_{A} \propto T^{8 / g-1 / 2}, \quad G_{A} \propto V^{8 / g-1 / 2}
$$

respectively for $e V<T$ and $e V>T$. Here and below we define the dimensionless conductance as $g=R_{q} / R$, where $R_{q}=h / e^{2}$ is the quantum resistance unit.

While the approach ${ }^{14}$ takes into account Coulomb interaction within a phenomenological scheme of the socalled $P(E)$-theory $\stackrel{15.16}{ }$, the question remains if and under which conditions this scheme is sufficient to account for the effect of electron-electron interactions in a disordered normal metal attached to a superconductor. In this paper we develop a microscopic analysis of this problem and evaluate the interaction correction to Andreev conductance of diffusive NS structures. We demonstrate that both the form and the magnitude of this interaction correction essentially depend on the dimensionality of the sample as well as on the relation between temperature and applied voltage to the relevant Thouless energy of the structure. In particular, we predict that at sufficiently low energies the interaction correction saturates to a constant value, thus providing a temperature- and voltage-independent renormalization of Andreev conductance. This saturation effect was recently observed ${ }^{17}$ in NS structures composed of a superconductor and multiwalled carbon nanotubes (MWNT).

The structure of the paper is as follows. In Sec. II we will define the model for the NS system and outline the formalism of a non-linear $\sigma$-model which will be used for our analysis. Sec. III contains the derivation of the general expression for the part of the action responsible for Andreev processes in our system. With the aid of this expression in Sec. IV we will find the Andreev current in the presence of electron-electron interactions. Its analysis in a number of important limiting cases is presented in Sec. V. In Sec. VI we will establish a general relation between the current and shot noise in the presence of electron-electron interactions. In Sec. VII we will briefly discuss our key observations and compare our results with recent experimental findings 17 . 


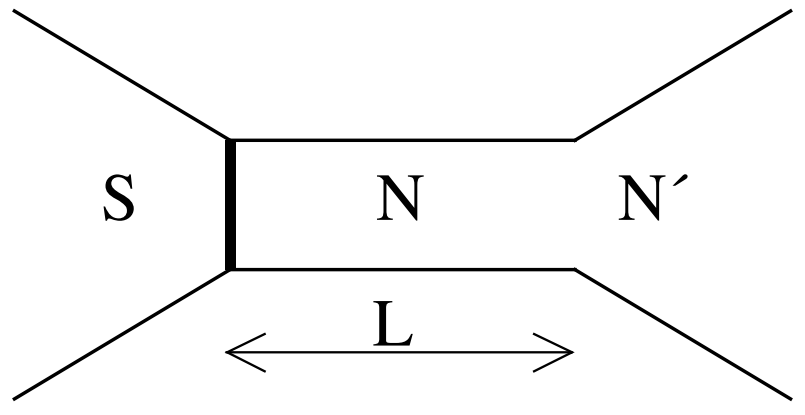

FIG. 1: NS hybrid structure under consideration.

\section{MODEL AND BASIC FORMALISM}

Throughout the paper we shall consider the hybrid structure depicted in Fig. 1. A piece of a normal metal of a simple rectangular shape and of length $L$ is attached to a bulk superconductor via an insulating interface and to a big metallic reservoir $\mathrm{N}^{\prime}$ via a highly conducting interface. The transversal dimensions of the normal metal are $L_{y}$ and $L_{z}$, hence, the cross-section of the NS interface $\Gamma$ is just the product $\Gamma=L_{y} L_{z}$. In what follows it will be convenient for us to assume that the transmission of a tunnel barrier at this interface is sufficiently low, so that its resistance $R_{t}$ exceeds that of a normal metal $R \equiv L / \sigma \Gamma$,

$$
R_{t} \gg R
$$

where $\sigma=2 e^{2} D N_{0}$ is the standard Drude conductivity with $e$ being the electron charge, $D=v_{F} l / 3$ standing for the diffusion coefficient, and $N_{0}$ representing the density of states per spin direction at the Fermi surface. In addition we will assume that the normal metal is shorter than the dephasing length $L_{\varphi}$,

$$
L \ll L_{\varphi} .
$$

Our theoretical analysis is based on the Keldysh representation of a non-linear $\sigma$-mode ${ }^{18,19}$ which is formulated in terms of the effective action

$$
S=S_{0}+S_{\Gamma}
$$

where

$$
\begin{aligned}
& S_{0}=\frac{i \pi N_{0}}{4} \operatorname{Tr}\left[D(\boldsymbol{\partial} \check{g})^{2}+4 i\left(i \check{\tau}_{z} \partial_{t}+\check{\Delta}-e \check{V}\right) \check{g}\right] \\
& S_{\Gamma}=-\frac{i \pi}{4 e^{2} R_{t} \Gamma} \operatorname{Tr}_{\Gamma} \check{g}_{-} \check{g}_{+}
\end{aligned}
$$

Here $\check{g}\left(\boldsymbol{R}, t_{1}, t_{2}\right)$ is the $4 \times 4$ matrix that depends on one coordinate and two time variables. It obeys the normalization condition

$$
\int d t^{\prime} \check{g}\left(\boldsymbol{R}, t_{1}, t^{\prime}\right) \check{g}\left(\boldsymbol{R}, t^{\prime}, t_{2}\right)=\delta\left(t_{1}-t_{2}\right) .
$$

The product of matrices $\check{g}$ in Eq. (6) should be understood as a convolution, cf. Eq. (8). The trace in Eq.
(6) is taken over the space and time variables and it is accompanied by the summation over matrix indices.

The term $S_{\Gamma}$ (7) follows directly from the KupriyanovLukichev boundary conditions $\mathrm{s}^{5}$ which account for tunneling of electrons between N- and S-metals. Spatial integration in this term is restricted to the insulating interface, subscripts $\mp$ denote matrices taken at the leftand right-hand sides of the interface. Other matrices in Eq. (6) are defined as follows

$$
\begin{aligned}
& \check{\tau}_{z}=\left(\begin{array}{cc}
\hat{\tau}_{z} & 0 \\
0 & \hat{\tau}_{z}
\end{array}\right), \quad \hat{\tau}_{z}=\left(\begin{array}{cc}
1 & 0 \\
0 & -1
\end{array}\right), \\
& \check{\Delta}(\boldsymbol{R}, t)=\left(\begin{array}{cc}
\hat{\Delta} & 0 \\
0 & \hat{\Delta}
\end{array}\right), \hat{\Delta}=\left(\begin{array}{cc}
0 & \Delta(\boldsymbol{R}, t) \\
-\Delta^{*}(\boldsymbol{R}, t) & 0
\end{array}\right) .
\end{aligned}
$$

The operator $\boldsymbol{\partial}$ acts as

$$
\begin{aligned}
& \boldsymbol{\partial} \check{g}=\nabla \check{g}-i \frac{e}{c}\left[\boldsymbol{A} \check{\tau}_{z}, \check{g}\right] \equiv \\
& \nabla \check{g}\left(t, t^{\prime}\right)-i \frac{e}{c} \boldsymbol{A}(t) \check{\tau}_{z} \check{g}\left(t, t^{\prime}\right)+\check{g}\left(t, t^{\prime}\right) i \frac{e}{c} \boldsymbol{A}\left(t^{\prime}\right) \check{\tau}_{z} \cdot
\end{aligned}
$$

Here $\boldsymbol{A}(\boldsymbol{R}, t)$ is the vector potential. In Eq. (10) spatial arguments are suppressed for brevity.

Finally, the $\check{V}$ term depends on the HubbardStratonovich fields $V_{1}$ and $V_{2}$ defined on the two branches of the Keldysh contour. These fluctuating fields emerge after the standard decoupling procedure in the term describing electron-electron interactions. We define

$$
\check{V}(\boldsymbol{R}, t)=\left(\begin{array}{cc}
V^{+\hat{1}} & \frac{1}{2} V^{-\hat{1}} \\
\frac{1}{2} V^{-\hat{1}} & V^{+\hat{1}}
\end{array}\right),
$$

where $\hat{1}$ is $2 \times 2$ unity matrix and $V^{+}=\left(V_{1}+V_{2}\right) / 2, V^{-}=$ $V_{1}-V_{2}$ define respectively "classical" and "quantum" fluctuating fields. If an external voltage $V$ is applied to the system, it should simply be added to the classical component $V^{+}$.

It is necessary to supplement the action (6), (7) describing fermionic degrees of freedom, by the action for the bosonic fields $V^{ \pm}$. The latter action determines the correlators for these fields which will be specified below.

\section{ANDREEV ACTION}

Let us first define the matrices $\check{g}_{ \pm}$in the absence of both electron-electron interactions and tunneling between metals. For the normal metal one has $\check{g}_{+}\left(\boldsymbol{R}, t, t^{\prime}\right)=\check{g}_{0}\left(t, t^{\prime}\right)$, where

$$
\begin{aligned}
& \check{g}_{0}=\left(\begin{array}{cc}
\hat{g}_{0}^{R} & \hat{g}_{0}^{K} \\
0 & \hat{g}_{0}^{A}
\end{array}\right), \quad \hat{g}_{0}^{R, A}\left(t, t^{\prime}\right)= \pm \hat{\tau}_{z} \delta\left(t-t^{\prime}\right), \\
& \hat{g}_{0}^{K}\left(t, t^{\prime}\right)=-\frac{2 i T}{\sinh \left[\pi T\left(t-t^{\prime}\right)\right]} \hat{\tau}_{z} .
\end{aligned}
$$

The Fourier transform of the Keldysh matrix $\hat{g}_{0}^{K}$ reads $\hat{g}_{0}^{K}(\epsilon)=2 \tanh [\epsilon / 2 T] \hat{\tau}_{z}$. In what follows we will consider the values of voltages and temperatures much smaller 
than the superconducting gap $e V, T \ll \Delta$. Hence, it will be sufficient for our purposes to specify the matrix $\check{g}_{-}$ for a superconducting electrode only at energies much smaller than $\Delta$. Under this condition we have

$$
\check{g}_{-}\left(t, t^{\prime}\right)=\left(\begin{array}{cccc}
0 & -i & 0 & 0 \\
i & 0 & 0 & 0 \\
0 & 0 & 0 & -i \\
0 & 0 & i & 0
\end{array}\right) \delta\left(t-t^{\prime}\right) .
$$

Let us turn on electron-electron interactions. While in a large superconducting electrode their influence on the matrix $\check{g}_{-}$can be safely neglected, the matrix $\check{g}_{+}$in the normal metal gets modified as $\frac{15,18}{}$

$$
\check{g}_{+}\left(\boldsymbol{R}, t_{1}, t_{2}\right)=e^{-i \check{K}\left(\boldsymbol{R}, t_{1}\right)} \check{g}_{0}\left(t_{1}, t_{2}\right) e^{i \check{K}\left(\boldsymbol{R}, t_{2}\right)} .
$$

Here the matrix $\check{K}$ has the structure

$$
\check{K}=\left(\begin{array}{cc}
\varphi^{+} \hat{\tau}_{z} & \varphi^{-} \hat{\tau}_{z} / 2 \\
\varphi^{-} \hat{\tau}_{z} / 2 & \varphi^{+} \hat{\tau}_{z}
\end{array}\right)
$$

and the phase fields $\varphi^{ \pm}$satisfy the equations

$$
\begin{aligned}
& \left(D q^{2}+i \omega\right) \varphi^{-}(\boldsymbol{q}, \omega)+e V^{-}(\boldsymbol{q}, \omega)=0, \\
& \left(D q^{2}-i \omega\right) \varphi^{+}(\boldsymbol{q}, \omega)-e V^{+}(\boldsymbol{q}, \omega)= \\
& -D q^{2} \varphi^{-}(\boldsymbol{q}, \omega) \operatorname{coth} \frac{\omega}{2 T} .
\end{aligned}
$$

One can easily check that the part of the variation of $S_{0}$ (6) linear both in $\check{g}$-variations and in the fields $V^{ \pm}$vanishes under the transformation (14)-(16). These equations are sufficient to account for the effect of Coulomb interactions for the problem in question.

Now let us include tunneling between metals into consideration. In order to eliminate the dependence of the matrix $\check{g}_{+}$on the fluctuating fields let us permute the factors $\exp ( \pm i \check{K})$ in the term $S_{\Gamma}$ (7) to act on the matrix $\check{g}_{-}$. After this transformation we obtain

$$
S_{\Gamma}=-\frac{i \pi}{4 e^{2} R_{t} \Gamma} \operatorname{Tr}_{\Gamma} \check{Q}_{-} \check{g}_{+},
$$

where

$$
\begin{aligned}
& \check{Q}_{-}\left(\boldsymbol{R}, t, t^{\prime}\right)=\left(\begin{array}{cc}
\hat{A}(\boldsymbol{R}, t) & \hat{B}(\boldsymbol{R}, t) \\
\hat{B}(\boldsymbol{R}, t) & \hat{A}(\boldsymbol{R}, t)
\end{array}\right) \delta\left(t-t^{\prime}\right), \\
& \hat{A}=\left(\begin{array}{cc}
0 & -i e^{2 i \varphi^{+}} \cos \varphi^{-} \\
i e^{-2 i \varphi^{+}} \cos \varphi^{-} & 0
\end{array}\right), \\
& \hat{B}=\left(\begin{array}{cc}
0 & e^{2 i \varphi^{+}} \sin \varphi^{-} \\
e^{-2 i \varphi^{+}} \sin \varphi^{-} & 0
\end{array}\right) .
\end{aligned}
$$

What remains is to evaluate the deviations of $\check{g}_{+}$from its normal state value (12) due to tunneling of Cooper pairs from the superconducting electrode into the normal metal. It is convenient to employ the following parameterization

$\mathcal{Q}=\check{u}_{\dot{g}} \check{u}, \quad \check{u}=\check{u}^{-1}=\left(\begin{array}{cc}\delta\left(t-t^{\prime}\right) \hat{1} & -\frac{i T}{\sinh \left[\pi T\left(t-t^{\prime}\right)\right]} \hat{1} \\ 0 & -\delta\left(t-t^{\prime}\right) \hat{1}\end{array}\right)$, which brings the matrix $\mathcal{Q}$ to the diagonal form in the absence of the proximity effect. The latter effect can can then be interpreted in terms of fluctuations of $\mathcal{Q}$ parameterized as

$$
\mathcal{Q}=e^{-\mathcal{W} / 2} \check{\sigma}_{z} \check{\tau}_{z} e^{\mathcal{W} / 2}=\check{\sigma}_{z} \check{\tau}_{z}\left(1+\mathcal{W}+\mathcal{W}^{2} / 2+\ldots\right)
$$

with

$$
\check{\sigma}_{z}=\left(\begin{array}{cc}
\hat{1} & 0 \\
0 & -\hat{1}
\end{array}\right) .
$$

The matrix $\mathcal{W}$ is in turn parameterized via the Pauli matrices $\hat{\tau}_{x, y, z}$ as

$$
\mathcal{W}=\left(\begin{array}{cc}
w_{1} \hat{\tau}_{x}+w_{2} \hat{\tau}_{y} & w_{0} \hat{1}+w_{3} \hat{\tau}_{z} \\
\bar{w}_{0} \hat{1}+\bar{w}_{3} \hat{\tau}_{z} & \bar{w}_{1} \hat{\tau}_{x}+\bar{w}_{2} \hat{\tau}_{y}
\end{array}\right) .
$$

The functions $w_{i}$, and $\bar{w}_{i}$ can be expanded in the eigenfunctions $\psi$ of the diffusion equation

$$
w_{i}(x, y, z)=\sum_{\boldsymbol{q}} w_{i}(\boldsymbol{q}) \psi_{\boldsymbol{q}}(x, y, z),
$$

obeying the boundary conditions $d \psi /\left.d x\right|_{x=0}=0$, $\left.\psi\right|_{x=L}=0$ and impenetrable boundary conditions at $y=0, L_{y}$ and $z=0, L_{z}$. We choose

$$
\begin{aligned}
& \psi_{\boldsymbol{q}}(x, y, z) \propto \cos q_{x} x \cos q_{y} y \cos q_{z} z \\
& q_{x}=\frac{\pi}{L}\left(l+\frac{1}{2}\right), q_{y}=\frac{\pi}{L_{y}} m, q_{z}=\frac{\pi}{L_{z}} n, \\
& l, m, n=0,1, \ldots
\end{aligned}
$$

The normalization constant is determined by the condition $\int d x d y d z \psi_{\boldsymbol{q}}^{2}(\boldsymbol{r})=1$.

Using the effective action (6), we can find all non-zero averages for the products of the functions $w_{i}$, and $\bar{w}_{i}$. Below we will only need the following averages 19

$$
\begin{aligned}
& \left\langle w_{i}\left(\boldsymbol{q}, \epsilon_{1}, \epsilon_{2}\right) w_{i}\left(-\boldsymbol{q}, \epsilon_{3}, \epsilon_{4}\right)\right\rangle= \\
& -\frac{1}{\pi N_{0}} \frac{(2 \pi)^{2} \delta\left(\epsilon_{1}-\epsilon_{4}\right) \delta\left(\epsilon_{2}-\epsilon_{3}\right)}{D q^{2}-i\left(\epsilon_{1}+\epsilon_{2}\right)}, \\
& \left\langle\bar{w}_{i}\left(\boldsymbol{q}, \epsilon_{1}, \epsilon_{2}\right) \bar{w}_{i}\left(-\boldsymbol{q}, \epsilon_{3}, \epsilon_{4}\right)\right\rangle= \\
& -\frac{1}{\pi N_{0}} \frac{(2 \pi)^{2} \delta\left(\epsilon_{1}-\epsilon_{4}\right) \delta\left(\epsilon_{2}-\epsilon_{3}\right)}{D q^{2}+i\left(\epsilon_{1}+\epsilon_{2}\right)},
\end{aligned}
$$

which correspond to the Cooperons $(i=1,2)$.

Now we are ready to evaluate the contribution of Andreev processes to the effective action. In the weak tunneling limit it is sufficient to $\operatorname{expand} \exp (i S)$ up to the second order in $S_{\Gamma}$ and then to average the resulting expression over the fermionic degrees of freedom with subsequent re-exponentiation of the result. In the limit $R_{t} \gg R$, the amplitude of the anomalous Green function penetrating into the normal metal remains much smaller than unity. Hence, in Eq. (20) it is sufficient to retain only the linear terms in $\mathcal{W}$. The resulting Andreev contribution to the action then takes the form

$$
\begin{aligned}
& \delta S_{A}=-\frac{i}{32}\left(\frac{\pi}{e^{2} R_{t} \Gamma}\right)^{2} \\
& \left\langle\operatorname{Tr}_{\Gamma_{1}} \mathcal{Q}_{-}\left(\Gamma_{1}\right) \check{\sigma}_{z} \check{\tau}_{z} \mathcal{W}\left(\Gamma_{1}\right) \cdot \operatorname{Tr}_{\Gamma_{2}} \mathcal{Q}_{-}\left(\Gamma_{2}\right) \check{\sigma}_{z} \check{\tau}_{z} \mathcal{W}\left(\Gamma_{2}\right)\right\rangle .
\end{aligned}
$$


The space integration is performed here along the same interface $\Gamma$, but independently for the terms containing $\Gamma_{1}$ and $\Gamma_{2}$. The matrix $\mathcal{Q}_{-}$is defined by the relation $\mathcal{Q}_{-}=\check{u} \check{Q}_{-} \check{u}$. Employing Eqs. (18), (19) and performing the averaging with the aid of Eqs. (25), we arrive at the final expression for $\delta S_{A}\left[\varphi^{ \pm}\right]$which will be extensively used in our subsequent analysis. We find

$$
\begin{aligned}
& \delta S_{A}=\frac{i \pi R D}{2 e^{2} R_{t}^{2} \Gamma L} \sum_{\boldsymbol{q}} \int \frac{d E d \omega}{(2 \pi)^{2}} \int_{\Gamma} d \boldsymbol{r} \int_{\Gamma} d \boldsymbol{r}^{\prime} \int d t \int d t^{\prime} e^{i \omega\left(t-t^{\prime}\right)} \psi_{\boldsymbol{q}}(\boldsymbol{r}) \psi_{\boldsymbol{q}}\left(\boldsymbol{r}^{\prime}\right) \times \\
& \left\{\frac { D q ^ { 2 } } { ( D q ^ { 2 } ) ^ { 2 } + 4 E ^ { 2 } } [ \operatorname { t a n h } \frac { E + ( \omega / 2 ) } { 2 T } - \operatorname { t a n h } \frac { E - ( \omega / 2 ) } { 2 T } ] \left[\operatorname{coth} \frac{\omega}{2 T} e^{-2 i\left(\varphi^{+}(\boldsymbol{r}, t)-\varphi^{+}\left(\boldsymbol{r}^{\prime}, t^{\prime}\right)\right)} \sin \left[\varphi^{-}(\boldsymbol{r}, t)\right] \sin \left[\varphi^{-}\left(\boldsymbol{r}^{\prime}, t^{\prime}\right)\right]\right.\right. \\
& \left.+\sin \left[2 \varphi^{+}(\boldsymbol{r}, t)-2 \varphi^{+}\left(\boldsymbol{r}^{\prime}, t^{\prime}\right)\right] \cos \left[\varphi^{-}(\boldsymbol{r}, t)\right] \sin \left[\varphi^{-}\left(\boldsymbol{r}^{\prime}, t^{\prime}\right)\right]\right] \\
& -\frac{2 i E}{\left(D q^{2}\right)^{2}+4 E^{2}}\left[\tanh \frac{E+(\omega / 2)}{2 T}+\tanh \frac{E-(\omega / 2)}{2 T}\right] \sin \left[2 \varphi^{+}(\boldsymbol{r}, t)-2 \varphi^{+}\left(\boldsymbol{r}^{\prime}, t^{\prime}\right)\right] \cos \left[\varphi^{-}(\boldsymbol{r}, t)\right] \sin \left[\varphi^{-}\left(\boldsymbol{r}^{\prime}, t^{\prime}\right)\right] \\
& \left.-\frac{D q^{2}}{\left(D q^{2}\right)^{2}+4 E^{2}} e^{2 i\left(\varphi^{+}(\boldsymbol{r}, t)-\varphi^{+}\left(\boldsymbol{r}^{\prime}, t^{\prime}\right)\right)} \cos \left[\varphi^{-}(\boldsymbol{r}, t)-\varphi^{-}\left(\boldsymbol{r}^{\prime}, t^{\prime}\right)\right]\right\}
\end{aligned}
$$

\section{ANDREEV CURRENT IN THE PRESENCE OF INTERACTIONS}

Let is first evaluate Andreev current across the NS interface. Substituting Eq. (27) into the formal expression for the current

$$
I=e\left\langle\int_{\Gamma} d^{2} r \frac{\delta}{\delta \varphi^{-}(\boldsymbol{r})} \delta S_{A}\right\rangle
$$

and performing averaging over the fluctuating fields $\varphi^{ \pm}$ we arrive at the general result for the Andreev current

$$
\begin{aligned}
& I=\frac{\pi R D}{2 e R_{t}^{2} \Gamma L} \sum_{\boldsymbol{q}} \int \frac{d E d \omega}{(2 \pi)^{2}} \int_{\Gamma} d^{2} r d^{2} r^{\prime} \psi_{\boldsymbol{q}}(\boldsymbol{r}) \psi_{\boldsymbol{q}}\left(\boldsymbol{r}^{\prime}\right)\left[\tanh \left(\frac{E+e V-(\omega / 2)}{2 T}\right)-\tanh \left(\frac{E-e V+(\omega / 2)}{2 T}\right)\right] \times \\
& \frac{D q^{2}}{\left(D q^{2}\right)^{2}+4 E^{2}} P\left(\omega, \boldsymbol{r}, \boldsymbol{r}^{\prime}\right) \frac{1-e^{-2 e V / T}}{1-e^{(\omega-2 e V) / T}} .
\end{aligned}
$$

Here the function

$$
\begin{aligned}
& P\left(\omega, \boldsymbol{r}, \boldsymbol{r}^{\prime}\right)=\int d t e^{J\left(t, \boldsymbol{r}, \boldsymbol{r}^{\prime}\right)+i \omega t}, \\
& e^{J\left(t, \boldsymbol{r}, \boldsymbol{r}^{\prime}\right)}=\left\langle e^{2 i \varphi_{2}(\boldsymbol{r}, t)-2 i \varphi_{1}\left(\boldsymbol{r}^{\prime}, 0\right)}\right\rangle
\end{aligned}
$$

accounts for the effect of interactions described by the fluctuating phase fields $\varphi_{1,2}=\varphi^{+} \pm\left(\varphi^{-} / 2\right)$ defined on the two branches of the Keldysh contour. Averaging in Eq. (30) is performed in the absence of the external voltage. Note that the form of this function is reminiscent to that used in the standard $P(E)$-theory ${ }^{15.16}$ and the result for the Andreev current (29) looks somewhat similar to the expression derived within the framework of the latter theory in Ref. 14. In contrast to the latter work, how- ever, our approach and resulting Eqs. (29), (30) fully account (i) for electron coherence across the whole NS structure and (ii) for the spatial dependence, introduced both by the functions $\psi_{\boldsymbol{q}}(\boldsymbol{r})$, describing fermionic fluctuations, and by the function $J\left(t, \boldsymbol{r}, \boldsymbol{r}^{\prime}\right)$, describing bosonic (phase) fluctuations.

These fluctuations depend on the dimensionality of the normal sample. For our purposes it will be sufficient to employ the standard random phase approximation (RPA) and to express the correlators of the fluctuating Hubbard-Stratonovich fields via an effective $d$-dimensional dielectric function $\varepsilon(\omega, k)$ of our system. 
One finds 20

$$
\begin{aligned}
& \left\langle e V^{+}(t, \boldsymbol{r}) e V^{+}(0,0)\right\rangle= \\
& -\int \frac{d \omega d^{d} k}{(2 \pi)^{d+1}} \operatorname{Im}\left(V^{*}(k, \omega)\right) \operatorname{coth}\left(\frac{\omega}{2 T}\right) e^{-i \omega t+i \boldsymbol{k} \boldsymbol{r}}, \\
& \left\langle e V^{+}(t, \boldsymbol{r}) e V^{-}(0,0)\right\rangle= \\
& i \int \frac{d \omega d^{d} k}{(2 \pi)^{d+1}} V^{*}(k, \omega) e^{-i \omega t+i \boldsymbol{k} \boldsymbol{r}}, \\
& \left\langle e V^{-}(t, \boldsymbol{r}) e V^{-}(0,0)\right\rangle=0 .
\end{aligned}
$$

Here we have defined

$$
V^{*}(k, \omega)=\frac{1}{V_{0}^{-1}(k)+\frac{k^{2}}{4 \pi e^{2}}[\varepsilon(k, \omega)-1]},
$$

where the term $V_{0}(k)$ stands for the unscreened Coulomb interaction:

$$
\begin{aligned}
V_{0}(k)=\frac{4 \pi e^{2}}{k^{2}}, & d=3, \\
V_{0}(k)=\frac{2 \pi e^{2}}{k}, & d=2, \\
V_{0}(k)=e^{2} \ln \left(1+\frac{1}{\Gamma k^{2}}\right), & d=1 .
\end{aligned}
$$

In the latter equation we have assumed $L_{y} \sim L_{z} \sim$ $\sqrt{\Gamma}$. In the three-dimensional case we obviously have $V^{*}(k, \omega)=V_{0}(k) / \varepsilon(k, \omega)$.

Note, that in the case of lower dimensions $d=1,2$ the term $V_{0}^{-1}(k)$ also accounts for the electric field energy outside the conductors. The corresponding interaction can be screened, provided there are metallic gates near the film/wire. For instance, in the case $d=2$ one has

$$
V_{0}^{s c r}(k)=\frac{2 \pi e^{2}}{k}\left(1-e^{-b k}\right),
$$

where $b$ is the distance between the film and the gate electrode.

In order to evaluate the average for the phase fields (30) one can combine Eqs. (16), (31) with the standard Drude form of the dielectric function for the normal metal

$$
\varepsilon(k, \omega)=1+\frac{4 \pi \sigma}{-i \omega+D k^{2}} .
$$

The resulting expressions for the function (30) obtained in this way remain applicable at frequencies above the corresponding Thouless energy of the sample but should fail at lower frequencies, in which case discrete wave vectors $\sim 1 / L$ become significant. In order to cure this complication it is useful to depart from the representation of plane waves in Eq. (31) and to employ a different set of the (volume-normalized) basis functions $\Omega_{m}(\boldsymbol{r})$ obeying the equation

$$
\nabla^{2} \Omega_{m}(\boldsymbol{r})+\lambda_{m}^{2} \Omega_{m}(\boldsymbol{r})=0
$$

with the corresponding eigenvalues $\lambda_{m}$. At the impenetrable interfaces the functions $\Omega_{m}(\boldsymbol{r})$ satisfy the boundary condition $\nabla_{n} \Omega_{m}(\boldsymbol{r})=0$. One should also require the functions $\Omega_{m}(\boldsymbol{r})$ to vanish at the interface with a large metallic reservoir $x=L$.

The corresponding generalization of the dielectric function (35) can be achieved by evaluating the response of a finite sample, characterized by the current density $\boldsymbol{j}(\boldsymbol{r})=\sigma \boldsymbol{E}(\boldsymbol{r})-D \nabla \rho(\boldsymbol{r})$ with $\boldsymbol{E}$ and $\rho$ standing respectively for the electric field and for the charge density. One $\operatorname{gets}^{21}$

$$
\begin{aligned}
& \varepsilon_{\alpha \beta}\left(\boldsymbol{r}, \boldsymbol{r}^{\prime}, \omega\right)=\delta_{\alpha \beta} \delta\left(\boldsymbol{r}-\boldsymbol{r}^{\prime}\right)+ \\
& \frac{4 \pi i}{\omega} \sigma_{D}\left(\delta_{\alpha \beta} \delta\left(\boldsymbol{r}-\boldsymbol{r}^{\prime}\right)-\nabla_{\alpha} \nabla_{\beta}^{\prime} d\left(\boldsymbol{r}, \boldsymbol{r}^{\prime}, \omega\right)\right),
\end{aligned}
$$

where

$$
d\left(\boldsymbol{r}, \boldsymbol{r}^{\prime}, \omega\right)=\sum_{m} \frac{\Omega_{m}(\boldsymbol{r}) \Omega_{m}\left(\boldsymbol{r}^{\prime}\right)}{\lambda_{m}^{2}-\frac{i \omega}{D}} .
$$

Implementing these modifications also into Eqs. (31) and employing Eqs. (16) we arrive at the following general result

$$
\begin{aligned}
& J\left(t, \boldsymbol{r}, \boldsymbol{r}^{\prime}\right)=4 \sum_{m} \int \frac{d \omega}{(2 \pi)} \operatorname{Im}\left[\frac{V^{*}\left(\lambda_{m}, \omega\right)}{\left(D \lambda_{m}^{2}-i \omega\right)^{2}}\right] \times \\
& {\left[\left(\operatorname{coth} \frac{\omega}{2 T} \cos \omega t-i \sin \omega t\right) \Omega_{m}(\boldsymbol{r}) \Omega_{m}\left(\boldsymbol{r}^{\prime}\right)-\right.} \\
& \left.\frac{1}{2} \operatorname{coth} \frac{\omega}{2 T}\left(\Omega_{m}(\boldsymbol{r}) \Omega_{m}(\boldsymbol{r})+\Omega_{m}\left(\boldsymbol{r}^{\prime}\right) \Omega_{m}\left(\boldsymbol{r}^{\prime}\right)\right)\right]
\end{aligned}
$$

The latter formula results in $J\left(t-(i / T), \boldsymbol{r}, \boldsymbol{r}^{\prime}\right)=$ $J\left(-t, \boldsymbol{r}, \boldsymbol{r}^{\prime}\right)$ leading to the relation $P\left(-E, \boldsymbol{r}, \boldsymbol{r}^{\prime}\right)=$ $e^{-E / T} P\left(\omega, \boldsymbol{r}, \boldsymbol{r}^{\prime}\right)$ as in the standard $P(E)$ theory. Accordingly, at $T \rightarrow 0$ Eq. (29) reduces to

$$
\begin{aligned}
& \frac{d I}{d V}=\frac{D R}{2 \pi R_{t}^{2} \Gamma L} \sum_{\boldsymbol{q}} \int_{0^{-}}^{2|e V|} d E \int_{\Gamma} d^{2} r d^{2} r^{\prime} \psi_{\boldsymbol{q}}(\boldsymbol{r}) \psi_{\boldsymbol{q}}\left(\boldsymbol{r}^{\prime}\right) \\
& P\left(E, \boldsymbol{r}, \boldsymbol{r}^{\prime}\right) \frac{D q^{2}}{\left(D q^{2}\right)^{2}+(2|e V|-E)^{2}} .
\end{aligned}
$$

At frequencies exceeding the sample Thouless energy it is possible to perform averaging of Eq. (39) over fluctuations depending on $\boldsymbol{r}+\boldsymbol{r}^{\prime}$. Then one gets

$$
\begin{aligned}
& J(t,|\boldsymbol{r}|)=4 \int \frac{d \omega d^{n} k}{(2 \pi)^{n+1}} \operatorname{Im}\left[\frac{\left.V^{*}(k, \omega)\right)}{\left(D k^{2}-i \omega\right)^{2}}\right] \times \\
& {\left[\operatorname{coth} \frac{\omega}{2 T}(\cos \omega t \cos \boldsymbol{k r}-1)-i \sin \omega t \cos \boldsymbol{k} \boldsymbol{r}\right]}
\end{aligned}
$$

\section{RESULTS}

\section{A. Non-interacting limit}

Let us first reproduce the results for Andreev current in the non-interacting limit. In this case we have $P(\omega, r)=$ 
$2 \pi \delta(\omega)$ and the standard expressions for the $I-V$ curve are readily recovered. In the limit $e V \gg T$ one finds

$$
\begin{aligned}
& \frac{d I}{d V}=\frac{R}{R_{t}^{2}}, \quad \text { if }|e V| \ll \epsilon_{\mathrm{Th}} ; \\
& \frac{d I}{d V}=\frac{R}{2 R_{t}^{2}} \sqrt{\frac{\epsilon_{\mathrm{Th}}}{|e V|}}, \quad \text { if }|e V| \gg \epsilon_{\mathrm{Th}} .
\end{aligned}
$$

Here and below $\epsilon_{\mathrm{Th}}=D / L^{2}$ is the Thouless energy.

In the linear regime $e V \ll T$ for the Andreev conductance $G_{A} \equiv d I / d V$ we obtain

$$
\begin{aligned}
& G_{A}^{(0)}=\frac{R}{R_{t}^{2}}, \quad \text { if } T \ll \epsilon_{\mathrm{Th}}, \\
& G_{A}^{(0)}=\frac{\left(2^{3 / 2}-1\right) \zeta(3 / 2)}{4 \sqrt{\pi}} \frac{R}{R_{t}^{2}} \sqrt{\frac{\epsilon_{\mathrm{Th}}}{T}}, \quad \text { if } T \gg \epsilon_{\mathrm{Th}},
\end{aligned}
$$

where $\left(2^{3 / 2}-1\right) \zeta(3 / 2) / 4 \sqrt{\pi} \approx 0.67$.

Now let us turn to the effect of electron-electron interactions. Both the value and the form of the interaction correction to the Andreev conductance essentially depend on the effective dimensionality of fluctuations of the phase fields. Depending on the voltage and temperature the leading contribution to the interaction correction is given either by the modes with $l, m, n \gg 1$ (cf. Eq. (24)) or by the modes with $m$ (or $n$, or both) being equal to zero. Correspondingly, below we will distinguish between effectively $3 \mathrm{~d}-$, quasi-2d- and quasi-1d-type of behavior of the interaction correction.

\section{B. Effect of interactions: Bulk limit}

We start from the case of $3 \mathrm{~d}$ fluctuations. For convenience we shall assume $L \gtrsim L_{y} \gtrsim L_{z}$ and consider the limit $e V \gg T$. At sufficiently high voltages $e V \gg D / L_{z}^{2}$ from Eq. (41) (with $d=3$ ) we obtain

$$
\begin{aligned}
& J(t, r)=\frac{e^{2}}{\pi^{2} \sigma r} \int_{0}^{\infty} \frac{\cos \left(2 D t \omega / r^{2}\right)}{\omega}\left(1-e^{-\sqrt{\omega}} \cos \sqrt{\omega}\right) d \omega \\
& -\frac{i e^{2}}{2 \pi \sigma r} \operatorname{erf}\left(\frac{r}{2 \sqrt{D|t|}}\right) \operatorname{sgn} t-\kappa
\end{aligned}
$$

where $\operatorname{erf}(x)=2 \int_{0}^{x} \exp \left(-t^{2}\right) d t / \sqrt{\pi}$ and

$$
\kappa=4 \int \frac{d \omega d^{3} k}{(2 \pi)^{4}} \operatorname{Im}\left[\frac{V^{*}(k, \omega)}{\left(D k^{2}-i \omega\right)^{2}}\right] \operatorname{sign} \omega .
$$

Making use of the condition $P(\omega, r)=0$ for $\omega<0$, we have for $\omega \geq 0$

$$
\begin{aligned}
& P(\omega, r)=e^{-\kappa} 2 \pi \delta(\omega)+ \\
& \frac{2 e^{2} e^{-\kappa}}{\pi \sigma r \omega}\left[1-e^{-r \sqrt{\omega / 2 D}} \cos (r \sqrt{\omega / 2 D})\right] .
\end{aligned}
$$

Thus we obtain

$$
\frac{d I}{d V}=\frac{R e^{-\kappa}}{2 R_{t}^{2}}\left(\sqrt{\frac{\epsilon_{\mathrm{Th}}}{|e V|}}+\frac{3.64 \Gamma}{g L^{2}}\right) .
$$

One observes that the relative "weight" of the second term in the brackets of (47) grows with voltage as $(1 / g) \sqrt{e V / \epsilon_{\mathrm{Th}}}$. This form is consistent with the standard square-root energy dependence of the interaction correction to the density of states. In the second term in Eq. (47) this dependence is exactly compensated by ZBA (42) resulting in the voltage-independent contribution $\sim R / g R_{t}^{2}$. This compensation will not occur in other limits to be considered below.

The value of $\kappa$ in the bulk limit can be estimated as

$$
\kappa \equiv \kappa_{3} \sim \frac{e^{2}}{\sigma \sqrt{D}} \int_{0}^{1 / \tau} \frac{d \omega}{\sqrt{\omega}} \sim \frac{1}{\left(p_{F} l\right)^{2}} .
$$

Here $\tau=l / v_{F}$ is the elastic scattering time.

While the result (47) holds at high voltages, at lower values $e V \lesssim \epsilon_{\mathrm{Th}}-$ as it was already discussed above the Andreev conductance saturates to a constant value

$$
\frac{d I}{d V}=\frac{R e^{-\kappa}}{R_{t}^{2}}
$$

with the voltage-dependent correction to this formula of order $(e V)^{2} / g \epsilon_{\text {Th }}^{2}$.

Note that in the bulk limit the net effect of electronelectron interactions is parametrically small and negative in the admissible range of voltages $\mathrm{eV}<1 / \tau$. Indeed, having in mind that our calculation is performed in the limit $p_{F} l \gg 1$ and making use of the estimate [48), in Eq. (47) one can expand $\exp (-\kappa)$ to the first order in $\kappa$ and observe that the corresponding negative correction exceeds the positive one by a factor $\sim 1 / \sqrt{|e V| \tau}$.

Let us also point out that in order to obtain the Andreev conductance in the voltage range $e V \ll T$ it is sufficient to simply substitute $T$ instead of $e V$. As in the non-interacting limit (cf. Eqs. (42) and (43)), this procedure will yield the correct form of the interaction correction up to an unimportant numerical prefactor of order one.

\section{Effect of interactions: Quasi-2d structures}

Let us now turn to low-dimensional fluctuations. We first consider systems which can be treated as quasi$2 \mathrm{~d}$ ones, i.e. we assume that both the length and the width of the normal metal strongly exceed its thickness $L \sim L_{y} \gg L_{z}$. We shall address two different physical situations corresponding to the absence and to the presence of massive metallic gate electrodes in the vicinity of the sample.

In the absence of the gate electrodes we can use the $2 \mathrm{~d}$ expression for $V_{0}(k)$ (33), combined with Eq. (32). We first evaluate the constant $\kappa$ which now consists of two contributions,

$$
\kappa=\kappa_{3}+\kappa_{2},
$$


where the first one is again determined by high frequencies $\left(\omega \gtrsim D / L_{z}^{2}\right)$ and small scales (cf. Eq. (48)), while the second one emerges from integrating over the parameter region $2 \pi \sigma L_{z} q \gg \omega$ and $D q^{2} \ll \omega$ in Eq. (39) for smaller frequencies $\omega \lesssim D / L_{z}^{2}$

$$
\kappa_{2}=\frac{8 L_{y}}{\pi g L} \ln \frac{L_{y}}{L_{z}} \ln \frac{\omega_{0} \Gamma}{D}, \quad \omega_{0}=\frac{\left(2 \pi \sigma L_{z}\right)^{2}}{D} .
$$

It is obvious from the above expressions that the contribution $\kappa_{3}$ exceeds $\kappa_{2}$ for thicker films, i.e. as long as $L_{z}$ remains bigger than the mean free path $l$. In the opposite case, however, the contribution of $3 \mathrm{~d}$ fluctuations $\kappa_{3}$ is negligible, and in the expression for $\kappa_{2}$ one should substitute $1 / \tau$ instead of $D / L_{z}^{2}$ in the arguments of the logarithms. Then one finds

$$
\kappa \simeq \kappa_{2}=\frac{2 L_{y}}{\pi g L} \ln \frac{L_{y}^{2}}{D \tau} \ln \frac{\omega_{0}^{2} \tau L_{y}^{2}}{D} .
$$

We observe that the above expressions for the renormalization constant $\kappa$ contain double logarithmic factors which are rather typical for $2 \mathrm{~d}$ structures. Similar double logarithmic dependencies emerge in voltage- and temperature-dependent contributions to be analyzed below.

Let us again stick to the limit $e V \gg T$. At voltages $D / L_{y}^{2} \ll e V \ll D / L_{z}^{2}$ we obtain

$$
\frac{d I}{d V}=\frac{R}{2 R_{t}^{2}} \sqrt{\frac{\epsilon_{\mathrm{Th}}}{|e V|}} e^{-\kappa}\left[1+\frac{2 L_{y}}{\pi g L} \ln \frac{|e V| L_{y}^{2}}{D} \ln \frac{\omega_{0}^{2} L_{y}^{2}}{|e V| D}\right]
$$

Note that in contrast to the expression for $\kappa$ (52) the voltage-dependent part of the interaction correction (53) does not involve the upper frequency cutoff $\sim 1 / \tau$. At lower voltages $e V \lesssim \epsilon_{\mathrm{Th}}$ the differential conductance again saturates to the value (49) with $\kappa$ defined in Eq. (52). The above results do not coincide with ones obtained in Ref. 19 for the same physical situation.

It is worth pointing out that even though the combination $L_{y} / g L$ in the above expressions always remains small, in some cases this smallness can be compensated by large logarithms, and the formal expansion in $1 / g$ can become insufficient. In this situation the $I-V$ curve can be evaluated with the aid of the following expression

$$
P(E)=2 \pi \delta(E) e^{-\Phi(0)}+2 \pi \frac{d}{d E} e^{-\Phi(E)},
$$

where

$$
\Phi(E)=\frac{e^{2}}{\pi^{2} \sigma L_{z}} \ln \frac{1}{\sqrt{\epsilon_{\mathrm{Th}}^{2}+E^{2}} \tau} \ln \frac{\omega_{0}^{2} \tau}{\sqrt{\epsilon_{\mathrm{Th}}^{2}+E^{2}}} .
$$

This function also enters into the expression for the density of states of normal $2 \mathrm{~d}$ films ${ }^{18}$

$$
N(\epsilon)=N_{0} e^{-\Phi(E) / 4} .
$$

We also note that in the case of thicker films $L_{z} \gg l$ our results are consistent with the expression for the density of $\operatorname{states}^{22}$.
In the presence of massive gate electrodes close to our sample the fluctuating electromagnetic fields do not extend outside its volume, and the term $V^{*}$ in Eq. (39) gets modified. In this limit we find

$$
\frac{d I}{d V}=\frac{R}{2 R_{t}^{2}} \sqrt{\frac{\epsilon_{\mathrm{Th}}}{|e V|}} e^{-\kappa}\left[1+\frac{2 L_{y}}{\pi g L} \ln ^{2} \frac{|e V| L_{y}^{2}}{D}\right],
$$

where

$$
\kappa=\frac{2 L_{y}}{\pi g L} \ln ^{2} \frac{L_{y}^{2}}{\tau D}
$$

If one considers the limit $T \gg e V$, it suffices to substitute $0.56 T$ instead of $e V$ in Eqs. (53), (57), and one will arrive at the expression for the linear Andreev conductance $G_{A}(T)$ valid with the logarithmic accuracy.

\section{Effect of interactions: Quasi-1d structures}

Let us finally turn to quasi-1d structures $L \gg L_{y} \sim L_{z}$ and examine the effect of electron-electron interactions on the Andreev conductance in the absence of screening gates. Similarly to the $2 \mathrm{~d}$ case, there also exists the parameter region $D q^{2} \ll \omega$ and $\sigma \Gamma q^{2} \log \left(1 / L_{z}^{2} q^{2}\right) \gg \omega$, which gives the leading contribution to $J(t)$. We obtain

$$
\begin{aligned}
& J(t)=16 \int_{\epsilon_{\mathrm{Th}}}^{\infty} \int_{q_{\mathrm{min}}}^{\infty} \frac{d q}{2 \pi} \frac{e^{2}}{\sigma \Gamma \omega q^{2}} \times \\
& \left\{\operatorname{coth} \frac{\omega}{2 T}[\cos (\omega t)-1]-i \sin (\omega t)\right\} .
\end{aligned}
$$

Since $1 / q$ cannot exceed $L$, one should choose

$$
q_{\min } \sim L^{-1}+\sqrt{\frac{\omega}{\sigma \Gamma \ln \left[\sigma \Gamma / \omega L_{z}^{2}\right]}} .
$$

Performing the $q$-integration one finds

$$
\begin{aligned}
& J(t)=\frac{8}{g} \int_{\epsilon_{\mathrm{Th}}}^{\infty} \frac{d \omega}{\omega(1+\sqrt{\omega R C})} \\
& \left\{\operatorname{coth} \frac{\omega}{2 T}[\cos (\omega t)-1]-i \sin (\omega t)\right\},
\end{aligned}
$$

where

$$
C=\frac{L}{\ln \left[L^{2} / \Gamma\right]}
$$

is the capacitance of a long cylinder. The expression (61) is rather similar to that obtained within the $P(E)$ theory ${ }^{14}$. There are at least two differences, however. One of them is that here the combination $1+\sqrt{\omega R C}$ enters in the denominator of Eq. (61), while in the mode ${ }^{14}$ one has $1+\omega^{2} R^{2} C_{J}^{2}$, where $C_{J}$ is the capacitance of a tunnel barrier at the NS interface. This capacitance is neglected in our analysis and can be trivially restored, if 


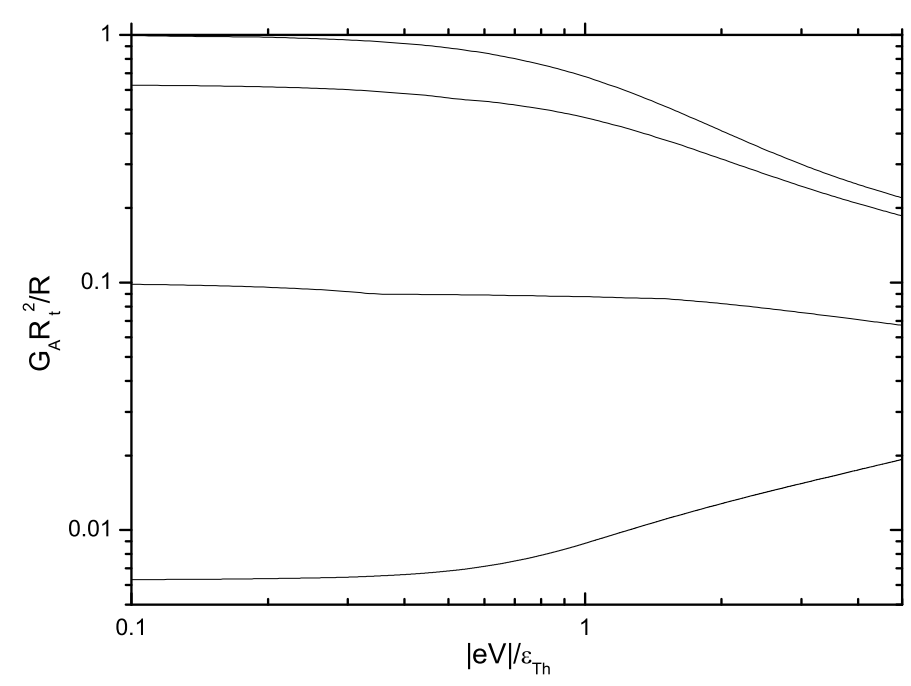

FIG. 2: The zero temperature differential Andreev conductance $G_{A}=d I / d V$ of quasi-1d diffusive NS systems. We have set $R C \epsilon_{\mathrm{Th}}=0.01$. The dimensionless parameter $8 / \mathrm{g}$ equals to $0,0.1,0.5,1.1$ (from top to bottom).

needed. Another - more important - difference is that the frequency integral in Eq. (61) cannot be extended below the Thouless energy. This low frequency cut-off results in the saturation of the interaction correction at small voltages and temperatures. This effect is not contained in the standard $P(E)$-theory which does not keep track of quantum coherence of electrons entering an effective environment and which also does not account for the sample geometry.

In the limit $T \rightarrow 0$ the asymptotic expression for the function $J(t)$ at $t \gg R C$ reads

$$
J(t)=\frac{8}{g}\left[\ln \left(\epsilon_{\mathrm{Th}} R C\right)-\mathrm{ci}\left(|t| \epsilon_{\mathrm{Th}}\right)+i \operatorname{sign} t \operatorname{si}\left(|t| \epsilon_{\mathrm{Th}}\right)\right],
$$

where $\operatorname{ci} t=-\int_{t}^{\infty} d z \cos z / z$, si $t=-\int_{t}^{\infty} d z \sin z / z$. In particular, for $t \ll 1 / \epsilon_{\mathrm{Th}}$ we get

$$
J(t)=-\frac{8}{g}\left[\ln \left(\frac{e^{\gamma} t}{R C}\right)+i \frac{\pi}{2} \operatorname{sign} t\right]
$$

where $\gamma \simeq 0.577 \ldots$ is the Euler constant. At longer times $t \gg 1 / \epsilon_{\mathrm{Th}}$ the function $J(t)$ approaches the constant, $J(t)=-\kappa$, where

$$
\kappa \simeq \kappa_{1}=\frac{8}{g} \ln \frac{1}{R C \epsilon_{\mathrm{Th}}}=\frac{8}{g} \ln \frac{e^{2} p_{F}^{2} \Gamma \ln \left(L^{2} / \Gamma\right)}{\pi^{2} v_{F}} .
$$

The $I-V$ curve can now be easily evaluated. It is clear from Eq. (61) that in the limit of high voltages $\mathrm{eV} \gg 1 / R C$ the response of an effective environment (normal metal) will be that of an $R C$-transmission line. In this limit the interaction correction to the Andreev conductance will behave analogously to that studied for single electron tunneling within the $P(E)$-theory $\underline{16}$. At

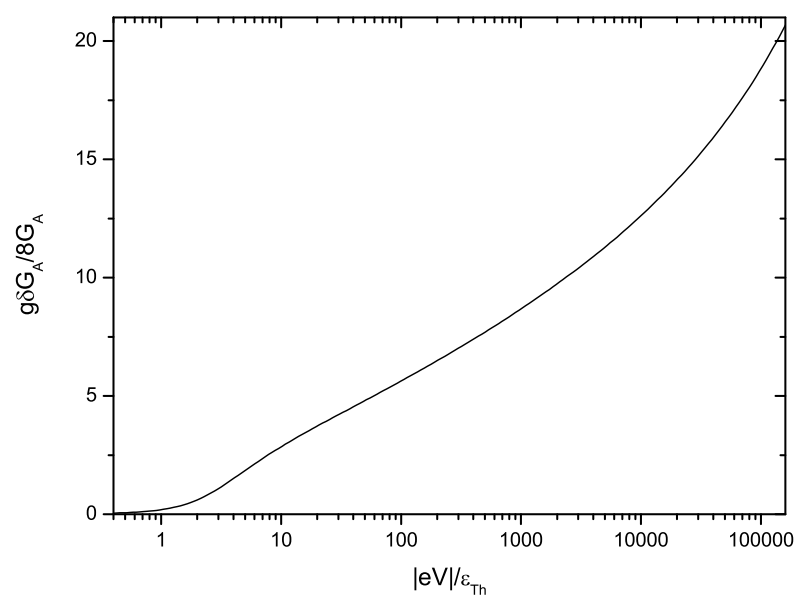

FIG. 3: The (normalized) value of the interaction correction to the conductance $\delta G_{A}=G_{A}-G_{A}^{(0)}$ as a function of voltage for $L_{y}=0.3 L, L_{z}=0.1 L$. One observes the saturation regime at $e V \lesssim \epsilon_{\mathrm{Th}}$, the $1 \mathrm{~d}$ logarithmic regime (67) at $\mathrm{eV} \gtrsim \epsilon_{\mathrm{Th}}$ which eventually crosses over to the $3 \mathrm{~d}$ regime (47) at $\mathrm{eV} \sim$ $10^{5} \epsilon_{\mathrm{Th}}$.

lower voltages $\epsilon_{\mathrm{Th}} \ll e V \ll 1 / R C$ we find

$$
\begin{aligned}
& \frac{d I}{d V}=\frac{R}{2 R_{t}^{2}} \sqrt{\frac{\epsilon_{\mathrm{Th}}}{|e V|}}\left[e^{-\kappa}+\right. \\
& \left.\frac{\sqrt{\pi} e^{-8 \gamma / g}}{\Gamma\left(\frac{8}{g}+\frac{1}{2}\right)}\left((2|e V| R C)^{8 / g}-\left(\epsilon_{\mathrm{Th}} R C\right)^{8 / g}\right)\right] .
\end{aligned}
$$

In the limit of large conductances $g \gg 1$ this expression can also be rewritten in a simpler form

$$
\frac{d I}{d V}=\frac{R}{2 R_{t}^{2}} \sqrt{\frac{\epsilon_{\mathrm{Th}}}{|e V|}} e^{-\kappa}\left[1+\frac{8}{g} \ln \frac{|e V|}{\epsilon_{\mathrm{Th}}}\right] .
$$

At smaller voltages $e V<\epsilon_{\mathrm{Th}}$ we again observe the saturation of the differential conductance to the value (49) where $\kappa$ is now determined by Eq. (65).

The above results remain practically the same also in the presence of screening electrodes in the vicinity of the sample. In that case for $g \gg 1$ we again recover the logarithmic correction (67) at large voltages while in the low voltage limit $e V \ll \epsilon_{\mathrm{Th}}$ we find

$$
\frac{d I}{d V}=\frac{R e^{-\kappa}}{R_{t}^{2}}\left[1+\frac{32}{15 g}\left(\frac{e V}{\epsilon_{\mathrm{Th}}}\right)^{2}\right] .
$$

As before, in order to obtain the linear Andreev conductance $G_{A}(T)$ in the regime $T \gg e V$ with the logarithmic accuracy, in the above expressions it suffices to substitute $0.56 T$ instead of $e V$.

The differential conductance of quasi-1d diffusive NS structures in the presence of electron-electron interactions is displayed in Fig. 2 for several values of the parameter $8 / g$. At larger voltages one observes a competition between two types of ZBA - conductance enhancement due to quantum interference of electrons $10,11,12,13$ 
and conductance suppression due to electron-electron interactions. For small conductances $g<16$ the second effect prevails and $d I / d V$ increases with voltage. At large conductances $g>16$ Coulomb effects become weaker and $d I / d V$ shows the opposite trend. For smaller voltages $\mathrm{eV}<\epsilon_{\mathrm{Th}}$ the Andreev conductance saturates to the voltage-independent value (49), (65) for all values of $g$. In this regime $d I / d V$ is always suppressed by electronelectron interactions and this effect becomes stronger with decreasing $g$.

Note, that the quasi-1d logarithmic behavior (67) is rather robust and can manifest itself for a wide range of voltages even if the sample length $L$ is not really much bigger than its width and thickness. An example of such a behavior is presented in Fig. 3 for the sample with $L_{y}=0.3 L, L_{z}=0.1 L$. In this particular case the logarithmic dependence of Eq. (67) remains applicable within a very wide voltage range $\epsilon_{\mathrm{Th}} \lesssim \mathrm{eV} \lesssim 10^{5} \epsilon_{\mathrm{Th}}$, while the $3 \mathrm{~d}$ regime (47) may set in only at huge voltages $e V>10^{5} \epsilon_{\mathrm{Th}}$.

\section{CURRENT NOISE}

Our effective action analysis also allows to study fluctuations of Andreev current in diffusive NS structures in the presence of electron-electron interactions. Here we restrict our attention to the current noise. The noise spectrum is expressed in terms of the current operators $\hat{I}$ in a standard way as

$$
\mathcal{S}\left(t, t^{\prime}\right)=\frac{1}{2}\left\langle\hat{I}(t) \hat{I}\left(t^{\prime}\right)+\hat{I}\left(t^{\prime}\right) \hat{I}(t)\right\rangle-\langle\hat{I}\rangle^{2} .
$$

This expression can easily be translated into

$\mathcal{S}\left(t, t^{\prime}\right)=-i e^{2}\left\langle\int_{\Gamma} d^{2} r \int_{\Gamma} d^{2} r^{\prime} \frac{\delta}{\delta \varphi^{-}(\boldsymbol{r}, t)} \frac{\delta}{\delta \varphi^{-}\left(\boldsymbol{r}^{\prime}, t^{\prime}\right)} \delta S_{A}\right\rangle$,

where $\delta S_{A}$ is defined in Eq. (27). Employing this general expression for the Andreev action together with the definitions of current and noise (28), (70) one can derive the following relationship

$$
\mathcal{S}(\omega, V, T)=e \sum_{ \pm} \operatorname{coth}\left(\frac{\omega \pm 2 e V}{2 T}\right) I\left(\frac{\omega}{2 e} \pm V, T\right),
$$

which remains valid irrespective of the dimensionality at frequencies and voltages smaller than both the inverse charge relaxation time $1 / R C$ and the inverse elastic scattering time $1 / \tau$.

Note that the analogous expression between the current and the noise spectrum was derived in Ref. 23 for normal tunnel barriers with interactions treated by means of the $P(E)$-theory and in Ref. 24 within a more general theoretical framework. Here Eq. (71) was derived for the Andreev current and the Andreev noise spectrum in diffusive NS structures in the presence of electronelectron interactions treated within a microscopic theory. Eq. (71) has exactly the same form as that ${ }^{23,24}$, one should only substitute $2 e$ instead of $e$ for the charge of the charge carriers. In the zero frequency limit Eq. (71) reduces to the generalized Schottky relation

$$
\mathcal{S}(\omega, V, T)=2 e \operatorname{coth}(e V / T) I(V, T),
$$

previously obtained in Ref. 25 in the absence of interactions.

Combining Eq. (71) with the results derived in the previous section one can fully describe the effect of Coulomb interaction on Andreev current noise in diffusive NS structures. Hence, there is no need to go into further details here. Let us only mention that - similarly to the Andreev conductance - at voltages/frequencies below $\epsilon_{\mathrm{Th}}$ we expect shot noise to be suppressed by the factor $\exp (-\kappa)$. At larger voltages $e V>\epsilon_{\mathrm{Th}}$ and in the low frequency limit the shot noise for quasi-1d NS structures should scale as

$$
\mathcal{S} \propto|V|^{8 / g-1 / 2} .
$$

\section{DISCUSSION}

The main observations of the present work can be summarized as follows. In hybrid NS structures electronelectron interactions generate two types of corrections to Andreev current. One of them is just a voltageindependent renormalization of the $I-V$ curve by the factor $\exp (-\kappa)$, while the other in general depends on voltage and/or temperature. The net effect of electronelectron interactions is always a reduction of the Andreev conductance. This reduction approaches its maximum value at temperatures and voltages below the Thouless energy of the sample. In this low energy regime the tunnel barrier and a normal metal act as a single coherent scatterer, whereas at energies above $\epsilon_{\mathrm{Th}}$ they can be treated as independent ones in the spirit of the $P(E)$ theory.

The magnitude of Coulomb effects essentially depends on the system dimensionality and is characterized by the dimensionless parameter $\kappa$. In $3 \mathrm{~d}$ systems we find $\kappa \sim 1 / p_{F}^{2} l^{2} \ll 1$, i.e. in this case Coulomb suppression of the Andreev conductance is weak except for very disordered samples. In the $2 \mathrm{~d}$ case $\kappa$ is proportional to the parameter $1 / p_{F}^{2} l L_{z}$ (which is again small) but is enhanced by the two logarithmic factors (cf. Eq. (52)), i.e. the net effect of electron-electron interactions is not necessarily weak in this case.

This effect becomes even more pronounced in quasi-1d systems in which case the value $\kappa$ is defined by Eq. (65). Obviously, the Coulomb suppression of the Andreev conductance can be very strong for such systems. Combining Eqs. (42), (43) and (49) with Eq. (65), and having in mind that for typical values of the Fermi velocity in metals one has $e^{2} / v_{F} \sim 1$, we arrive at a very simple estimate for the Andreev conductance in the presence of electron-electron interactions:

$$
G_{A} / G_{A}^{(0)} \sim 1 / N_{\mathrm{Ch}}^{8 / g},
$$


where $N_{\mathrm{Ch}} \sim p_{F}^{2} \Gamma$ is the number of conducting channels in the normal wire. In Eq. (74) for simplicity we have disregarded an additional weak (logarithmic) dependence on $L$ and $\Gamma$, cf. Eq. (65). Within this accuracy, Eq. (74) demonstrates that Coulomb suppression of the Andreev conductance in quasi-1d diffusive samples is determined by the number of conducting channels to the power $8 / \mathrm{g}$. We believe this prediction can be directly tested in future experiments.

Recently the authors ${ }^{17}$ have experimentally investigated the $I-V$ curves for hybrid systems composed of multi-walled carbon nanotubes (MWNT) attached to a superconducting electrode. The measurements $\frac{17}{17}$ performed below the superconducting critical temperature $T_{C}$ have revealed power-law dependencies of the differential conductance on voltage and temperature $d I / d V \propto$ $T^{\alpha_{S}}$ and $d I / d V \propto V^{\alpha_{S}}$. At smaller voltages $V<V_{\text {sat }} \sim$ $1 \mathrm{mV}$ the conductance was found to saturate to a constant value. Also at $T>T_{C}$ the power-law dependencies have been observed $d I / d V \propto T^{\alpha_{N}}$ with the value $\alpha_{N}$ somewhat smaller than $\alpha_{S}$.

It is interesting to compare our theoretical predictions with these experimental results. The effect of conductance saturation observed in Ref. 17 just matches with our prediction (74) and the value $e V_{\text {sat }}$ appears to be in a good agreement with the Thouless energy $\epsilon_{\mathrm{Th}}$ estimated for MWNT 17 . Also the observed power-law dependencies are consistent with our theoretical picture. Identifying $\alpha_{S}=8 / g-1 / 2$ and making use of the experimental value $\alpha_{S} \simeq 1.28$, we can estimate the effective dimension- less conductance for MWNT as $g \approx 4.5$. Above $T_{C}$ for the superconducting electrode one also expects the power-law dependence of the conductance on temperature15.16. Neglecting the impedance of the bulk electrode one would trivially get $\alpha_{N}=2 / g \approx 0.45$ which is slightly smaller than the measured values $\alpha_{N} \approx 0.77$ close to $T_{C}$ and $\alpha_{N} \approx 0.55$ at higher temperatures. It is clear, however, that above $T_{C}$ the impedance of the superconducting electrode $R_{S}$ becomes non-zero and should also be taken into account. Accordingly, the value $\alpha_{N}$ should be modified as $\alpha_{N}=2 / g+2 / g_{S}$, where $g_{S}=R_{q} / R_{S}$. This additional contribution to $\alpha_{N}$ could further improve the quantitative agreement between our theory and the experimental observations 17 .

Finally, we would like to point out that it would also be interesting to experimentally study the effect of Coulomb interactions on the shot noise in quasi-1d hybrid NS systems, like, e.g., ones investigated in Ref. 17. According to the results obtained in Sec. VI, at low voltages one expects suppression of the shot noise by the factor $\sim N_{\mathrm{Ch}}^{8 / g}$ while at higher voltages the power-law dependence (73) should be recovered. Simultaneous current and shot noise measurements could help to experimentally verify the general relation (71) between the noise spectrum and Andreev current in hybrid NS structures.

\section{Acknowledgments}

We are grateful to D.S. Golubev for useful discussions. We also thank the authors of Ref. 17 for communicating their results to us prior to publication.
1 A.F. Andreev, Zh. Eksp. Teor. Fiz. 46, 1823 (1964) [Sov. Phys. JETP 19, 1228 (1964)].

2 P.G. de Gennes, Superconductivity of Metals and Alloys (Benjamin, New York, 1966).

3 L.G. Aslamazov, A.I. Larkin, and Yu.N. Ovchinnikov, Zh. Eksp. Teor. Fiz. 55, 323 (1968) [Sov. Phys. JETP 28, 171 (1968)]

4 A.D. Zaikin and G.F. Zharkov, Fiz. Nizk. Temp. 7, 375 (1981) [Sov. J. Low Temp. Phys. 7, 181 (1981)].

5 M.Yu. Kupriyanov and V.F. Lukichev, Zh. Eksp. Teor. Fiz. 94, 139 (1988) [Sov. Phys. JETP 67, 1163 (1988)].

6 P. Dubos, H. Courtois, B. Pannetier, F.K. Wilhelm, A.D. Zaikin, and G. Schön, Phys. Rev. B 63, 064502 (2001).

7 A.D. Zaikin. Solid State Commun. 41, 533 (1982).

8 W. Belzig, C. Bruder, and G. Schön, Phys. Rev. B 53, 5727 (1996).

9 G.E. Blonder, M. Tinkham, and T.M. Klapwijk, Phys. Rev. B 25, 4515 (1982).

10 A.F. Volkov, A.V. Zaitsev, and T.M. Klapwijk, Physica C 210, 21 (1993).

11 F.W.J. Hekking and Yu.V. Nazarov, Phys. Rev. Lett. 71, 1625 (1993); Phys. Rev. B 49, 6847 (1994).

12 C.W.J. Beenakker, B. Rejaei, and J.A. Melsen, Phys. Rev. Lett. 72, 2470 (1994).

13 A.D. Zaikin, Physica B 203, 255 (1994).
14 A. Huck, F.W.J. Hekking, and B. Kramer, Europhys. Lett. 41, 201 (1998).

15 G. Schön and A.D. Zaikin, Phys. Rep. 198, 237 (1990).

16 G.-L. Ingold and Yu.V. Nazarov, in Single Charge Tunneling, ed. by H. Grabert and M. Devoret, NATO ASI Series B, vol. 294, pp. 21-107 (Plenum, 1992).

17 I. Takesue, T. Akazaki, S. Miyadai, N. Kobayashi, A. Tokita, M. Nomura, J. Haruyama, and H. Takayanagi, Physica E 24, 32 (2004).

18 A. Kamenev and A. Andreev, Phys. Rev. B 60, 2218 (1999).

19 M.V. Feigel'man, A.I. Larkin, and M.A. Skvortsov, Phys. Rev. B 61, 12361 (2000).

20 D.S. Golubev and A.D. Zaikin, Phys. Rev. B 59, 9195 (1999).

21 R.A. Serota and B. Goodman, Mod. Phys. Lett. B 13, 649 (1999).

22 J. Rollbühler and H. Grabert, Phys. Rev. Lett. 87, 126804 (2001).

${ }^{23}$ H. Lee and L.S. Levitov, Phys. Rev. B 53, 7383 (1996).

24 E.V. Sukhorukov, G. Burkard, and D. Loss, Phys. Rev. B 63, 125315 (2001).

25 F. Pistolesi, G. Bignon, and F.W.J. Hekking, Phys. Rev. B 69, 214518 (2004). 